\title{
Reducing Viability Bias in Analysis of Gut Microbiota in Preterm Infants at Risk of NEC and Sepsis
}

\author{
Gregory R. Young ${ }^{1 *}$, Darren L. Smith ${ }^{1}$, Nicholas D. Embleton ${ }^{2}$, Janet E. Berrington ${ }^{2}$, \\ Edward C. Schwalbe ${ }^{1}$, Stephen P. Cummings ${ }^{3}$, Christopher J. van der Gast ${ }^{4}$ and \\ Clare Lanyon ${ }^{1 *}$ \\ ${ }^{1}$ Faculty of Health and Life Sciences, University of Northumbria, Newcastle upon Tyne, United Kingdom, ${ }^{2}$ Newcastle \\ Neonatal Service, Newcastle upon Tyne Hospitals NHS Foundation Trust, Newcastle upon Tyne, United Kingdom, ${ }^{3}$ School of \\ Science and Engineering, Teesside University, Middlesbrough, United Kingdom, ${ }^{4}$ School of Healthcare Science, Manchester \\ Metropolitan University, Manchester, United Kingdom
}

\section{OPEN ACCESS}

Edited by:

Nathan W. Schmidt, University of Louisville, United States

Reviewed by:

Valerio lebba

Sapienza Università di Roma, Italy Renate Lux,

UCLA School of Dentistry, United States

*Correspondence:

Gregory R. Young

greg.young@northumbria.ac.uk

Clare Lanyon

clare.lanyon@northumbria.ac.uk

Received: 17 March 2017 Accepted: 22 May 2017

Published: 06 June 2017

Citation:

Young GR, Smith $D L$, Embleton $N D$,

Berrington JE, Schwalbe EC,

Cummings SP, van der Gast CJ and Lanyon C (2017) Reducing Viability Bias in Analysis of Gut Microbiota in Preterm Infants at Risk of NEC and Sepsis.

Front. Cell. Infect. Microbiol. 7:237. doi: 10.3389/fcimb.2017.00237
Necrotising enterocolitis (NEC) and sepsis are serious diseases of preterm infants that can result in feeding intolerance, the need for bowel resection, impaired physiological and neurological development, and high mortality rates. Neonatal healthcare improvements have allowed greater survival rates in preterm infants leading to increased numbers at risk of developing NEC and sepsis. Gut bacteria play a role in protection from or propensity to these conditions and have therefore, been studied extensively using targeted 16S rRNA gene sequencing methods. However, exact epidemiology of these conditions remain unknown and the role of the gut microbiota in NEC remains enigmatic. Many studies have confounding variables such as differing clinical intervention strategies or major methodological issues such as the inability of $16 \mathrm{~S}$ rRNA gene sequencing methods to determine viable from non-viable taxa. Identification of viable community members is important to identify links between the microbiota and disease in the highly unstable preterm infant gut. This is especially important as remnant DNA is robust and persists in the sampling environment following cell death. Chelation of such DNA prevents downstream amplification and inclusion in microbiota characterisation. This study validates use of propidium monoazide (PMA), a DNA chelating agent that is excluded by an undamaged bacterial membrane, to reduce bias associated with $16 S$ rRNA gene analysis of clinical stool samples. We aim to improve identification of the viable microbiota in order to increase the accuracy of clinical inferences made regarding the impact of the preterm gut microbiota on health and disease. Gut microbiota analysis was completed on stools from matched twins $(n=16)$ that received probiotics. Samples were treated with PMA, prior to bacterial DNA extraction. Meta-analysis highlighted a significant reduction in bacterial diversity in $68.8 \%$ of PMA treated samples as well as significantly reduced overall rare taxa abundance. Importantly, overall abundances of genera associated with protection from and propensity to NEC and sepsis such as: Bifidobacterium; Clostridium, and Staphylococcus sp. were significantly different following PMA-treatment. These results suggest non-viable cell exclusion by PMA-treatment reduces bias in gut microbiota analysis from which clinical inferences regarding patient susceptibility to NEC and sepsis are made.

Keywords: preterm, neonate, stool, microbiota, viability, propidium monoazide 


\section{INTRODUCTION}

Severely preterm infants ( $<32$ weeks) have immature immune systems (Levy, 2007; Strunk et al., 2011), improperly formed intestinal lumen (Halpern and Denning, 2015), and often feeding intolerance (Fanaro, 2013). All such characteristics increase the risk of onset of nosocomial infection, necrotising enterocolitis (NEC), and sepsis (Gregory et al., 2011). Outbreaks of NEC within the neonatal intensive care unit (NICU) (Boccia et al., 2001) and the absence of such diseases prior to bacterial colonization at birth suggest a key role of gut bacterial dysbiosis in these conditions. True causation is, however, very difficult to identify and compacted by complex to understand, highly turbulent community characteristics in the gut, probably in part affected by "routine" interventions of neonatal intensive care (antibiotic administration, feeding strategies, etc.) (Stoll et al., 1996; Hoy et al., 2000).

Targeted 16S rRNA gene sequencing technologies are used to produce microbial metadata for entire populations of a biotope with superior depth, specificity and, most importantly, in significantly less time than previous culture-based or molecular methods (Weinstock, 2012). In addition, the price of sequencing continues to decline (Caporaso et al., 2012), providing further incentives for microbiologists to employ this technique. However, $16 \mathrm{~S}$ rRNA gene sequencing does introduce inherent biases (von Wintzingerode et al., 1997), including enrichment of particular bacterial groups before storage (Rochelle et al., 1994), insufficient or preferential disruption of certain bacterial cells (Leff et al., 1995; Schneegurt et al., 2003), introduction of sequencing artefacts such as chimeras (Wang and Wang, 1996) and inability to exclude DNA from non-viable sources (Nocker et al., 2006; Nocker and Camper, 2009; Rogers et al., 2013). Persistence of non-viable DNA is due to the stability of the molecule, which enables DNA to remain in an environment long after the originating organism has died. DNA from non-viable bacterial cells (NVBCs) can persist in the lumen of the GI tract, resulting in identification during targeted 16S rRNA gene sequencing analyses. Such bias is especially important whilst studying the highly unstable (Koenig et al., 2011; Bergstrom et al., 2014), low diversity (Tuddenham and Sears, 2015) gut bacterial communities of severely preterm infants. A technique to enable non-viable cell exclusion (NVCE), from such analyses is, therefore, an important and necessary requirement in order to reduce bias and improve the current understanding of bacterial taxa associated with NEC and sepsis.

PMA is a DNA chelating compound that cannot translocate across a viable cellular membrane (Nocker et al., 2007). Nocker et al. (2006), developed the use of Propidium Monoazide (PMA), for differentiation between viable and non-viable bacterial cells during targeted 16S rRNA gene sequencing microbiota analyses (Nocker et al., 2006; Nocker and Camper, 2009; Rogers et al., 2013). This process has been applied in microbial ecology studies of other environments, including wastewater samples (Nocker et al., 2007, 2010), human oral cavities (Sanchez et al., 2013), human adult faeces (Bae and Wuertz, 2009; Fujimoto and Watanabe, 2013), the cystic fibrosis lung (Rogers et al., 2010; Nguyen et al., 2016), and other lower lung respiratory infections
(Rogers et al., 2013). The technique, however, has not yet been validated for use in the unique biotope of preterm infant stool despite vast quantities of research being published regarding this microbiota (Mshvildadze et al., 2010; Mai et al., 2011; Torrazza et al., 2013; McMurty et al., 2015). Furthermore, no studies so far have validated combining PMA treatment of this sample type in conjunction with the Schloss method for paired end targeted 16S rRNA gene sequencing (Kozich et al., 2013).

This study aims to identify and alleviate the bias associated with non-viable bacterial DNA inclusion in studies of the gut microbiota of significantly preterm infants at risk of NEC and sepsis. In doing so we hope to increase the accuracy of microbiota characterization in patients at risk of NEC and sepsis, therefore improving the quality of clinical inferences made in relation to the conditions.

The effects of PMA treatment were assessed by comparing bacterial richness, diversity, and community structure as well as individual taxa abundances within PMA-treated and untreated frozen stool samples $(n=16)$ when assessed using targeted paired end sequencing of the $16 \mathrm{~S}$ rRNA gene.

\section{MATERIALS AND METHODS}

Faecal samples were collected when available from day of life 43-81 from a set of significantly preterm twins born $25(+2)$ weeks gestation and at $\leq 710 \mathrm{~g}$, enrolled on the SERVIS study at the Royal Victoria Infirmary NICU, Newcastle upon Tyne, England, with ethical permission (NRES Committee North East-Newcastle \& North Tyneside 2). Both patients were administered Infloran ${ }^{\circledR}$ (Laboratorio Farmaceutico SIT, Mede, PV, ITA) probiotic supplements throughout the course of the sampling period (Bifidobacterium bifidum, Lactobacillus acidophilus). Stool was collected in sterile glass pots with sealed lids and frozen immediately on the ward. Batch collection and transportation to freezers at Northumbria University followed. Samples were stored at $-80^{\circ} \mathrm{C}$ until PMA treatment and DNA extraction for analysis.

\section{PMA Treatment and DNA Extraction}

PMA was supplied by Biotium (Hayward, CA, USA), and dissolved in dimethyl sulfoxide to a stock concentration of $20 \mathrm{mM}$. Faecal samples were homogenised in $2.5 \mathrm{ml}$ PBS per $0.1 \mathrm{~g}$ of stool $(\leq 0.5 \mathrm{~g})$, and centrifuged. The centrifuged pellet was resusupended in $2 \mathrm{ml} \mathrm{PBS}$ and split evenly to facilitate PMA-treated and untreated conditions per sample. PMA stock solution was added to a final concentration of $50 \mu \mathrm{M}$ in treated samples and the equivalent volume of PBS was added to untreated samples. PMA cross-linking was initiated by $30 \mathrm{~min}$ incubation on ice, in the dark with occasional mixing. Following this, samples were exposed to blue LED light at $464 \mathrm{~nm}$ during 30-s intervals for a total of $2 \mathrm{~min}$. After light exposure, samples were centrifuged at 10,000 $\times \mathrm{g}$ for $5 \mathrm{~min}$. The supernatant was discarded and DNA extracted from the cellular pellet using MoBio PowerLyzer PowerSoil DNA Isolation Kit (Carlsbad, CA, USA), as per manufacturer's instructions. 


\section{Nested PCR Protocol and MiSeq Analysis}

Prior to paired end targeted 16S rRNA gene analysis, extracted viable DNA was amplified by PCR. Nested PCR was employed in this scenario not to increase copy number prior to sequencing but to increase impact of PMA-intercalation of DNA by blocking amplification of the whole $16 \mathrm{~S}$ rRNA gene sequence prior to targeted sequencing of the shorter V4 region. Banihashemi et al. (2012) showed that amplification of a $200 \mathrm{bp}$ fragment failed to omit dead cell signals fully from DNA based community analyses. Universal bacterial 16S rRNA gene specific primers 27f (Lane, 1991), and 1,492r (Turner et al., 1999) were used under the following conditions: initial denaturation at $95^{\circ} \mathrm{C}$ for $5 \mathrm{~min}$ then 25 cycles of $30 \mathrm{~s}$ denaturation at $95^{\circ} \mathrm{C}$; primer annealing at $44.5^{\circ} \mathrm{C}$ for $30 \mathrm{~s}$; elongation at $72^{\circ} \mathrm{C}$ for $30 \mathrm{~s}$ then a final elongation at $72^{\circ} \mathrm{C}$ for $10 \mathrm{~min}$.

PCR products were serially diluted 1:10 and paired end targeted analysis of V4 regions of the 16S rRNA gene was performed as described by Kozich et al. (2013), on the Illumina MiSeq using primers described by Caporaso et al. (2011). MiSeq $250 \times 2$ chemistry was used to perform the targeted $16 \mathrm{~S}$ rRNA sequencing.

\section{Analysis}

Sequence reads with phred-score $\geq \mathrm{Q} 30$ were trimmed, merged and processed in Mothur (Schloss et al., 2009), following the MiSeq SOP. Number of sequences passing Q30 in each sample are illustrated in Figure S1. Reads with phred-score $<$ Q30 were not included in analysis. Uncorrected pairwise distances were calculated before clustering sequences in to OTUs using average neighbor joining, as recommended by Schloss and Westcott (2011). The same sequence reads were also submitted to the EBI ENA database for analysis (study accession PRJEB10326; http://www.ebi.ac.uk/ena/data/view/PRJEB10326).

Singletons were not removed from analysis to allow identification of PMA-treatment on all rare taxa identified by targeted sequencing. Normalization was not performed by rarefaction or subsampling due to the nature of the investigation. Instead relative abundances of individual taxa per sample were calculated. This is because the impact of PMA NVCE was assessed by omission of sequence reads from the community, therefore the absence of any sequence read was as informative as the presence of the same.

Per sample richness and beta-diversity was calculated using R statistical software (R_Core_Team., 2014) and the vegan package for community ecology (Oksanen et al., 2015). Metaanalysis (Borenstein et al., 2009) was used to compare results by treatment condition. Meta-analysis has previously been used to quantify the effect of PMA-treatment on bacterial communities of expectorated CF sputum samples (Rogers et al., 2013), allowing direct comparison of the effect of PMA-treatment between paired and unpaired samples by comparing effect size, rather than comparing means of highly variable individual samples by $t$-test. Each microbiota was randomly sub-sampled with bootstrapping $n=1,000$ times. Standard error was reported.

SIMPER comparison of individual taxa relative abundance per treatment condition was performed using PAST (Hammer et al., 2001). Significance of results was calculated and plotted using $R$ statistical software.
Comparison of non-frozen and frozen stool microbiotas was performed using ANOSIM and unconstrained Morisita-Horn cluster analysis.

\section{RESULTS}

Stool samples from a set of significantly preterm twins $(25+2$ weeks gestation) $(n=16)$ receiving Infloran ${ }^{\circledR}$ probiotic supplements were subjected to PMA-treatment for comparison to an untreated control of each sample. 16S rRNA gene sequencing identified a total of 161 individual taxa producing $4.72 \times 10^{6}$ total reads from $16 \times 2$ samples.

\section{Identification of Common and Rare Taxa}

To identify differences between common and rare taxa in PMA-treated and untreated conditions distribution abundance relationship plots were produced (Figure 1).

Significant positive distribution abundance relationships were observed between taxa abundance and persistence of taxa across samples in both treatment conditions (untreated: $r^{2}=0.58, n=$ 120, $P=<0.001$; PMA-treated: $\left.r^{2}=0.72, n=97, P=<0.001\right)$. Using this relationship, taxa in the upper quartile of occupancy ( $\geq 75 \%$ samples), in each treatment condition were classified as common, the remaining taxa were classified as rare.

Distribution of taxa appeared more even in the PMA-treated condition: fewer ubiquitous taxa dominate the communities in the PMA-treated condition (2 taxa); compared to the untreated condition (6 taxa).

In untreated sample conditions 6 taxa were identified as common, all of which were observed in every sample. Bifidobacterium, Enterococcus, 2 Clostridia spp., a Veillonella and an unclassified Enterobacteriaceae accounted for $77.9 \%$ of the total community member sequences. In PMA-treated samples, 8 common taxa were identified, comprising $82.2 \%$ of total community member sequences however of these, only 2 (Bifidobacterium and Enterococcus) were found in all samples. Anaerococcus and Finegoldia sp. were identified as common in PMA-treated samples but not in untreated samples.

\section{Effect of PMA Treatment on Bacterial Richness and Diversity}

Due to the large coverage variability between the stool sample communities $\left(m=1.47 \times 10^{5}, S D=1.26 \times 10^{5}\right)$, metaanalysis was used to identify the effect size of PMA-treatment on microbiota composition by bacterial OTU richness $(\mathrm{O} *)$; Shannon diversity index $\left(\mathrm{H}^{\prime}\right)$; and Inverse Simpson's diversity index (1/D).

Bacterial OTU richness was variable between stool samples of the same treatment condition (untreated $m=9.1 \pm 2.7$, PMAtreated $m=8.8 \pm 1.9$ ). The effect of PMA-treatment on $\mathrm{O} *$ was only once greater than the significance threshold (0.2), and showed no directional consistency (Figure 2).

Like richness, bacterial diversity also varied between individual stool samples in the same treatment condition (Table 1).

Meta-analysis showed negative effect sizes of PMA-treatment on bacterial diversity in $71.9 \%$ of samples, of which $73.9 \%$ were highly significant $(>0.8)$ (Figures 3,4). Significant negative mean 


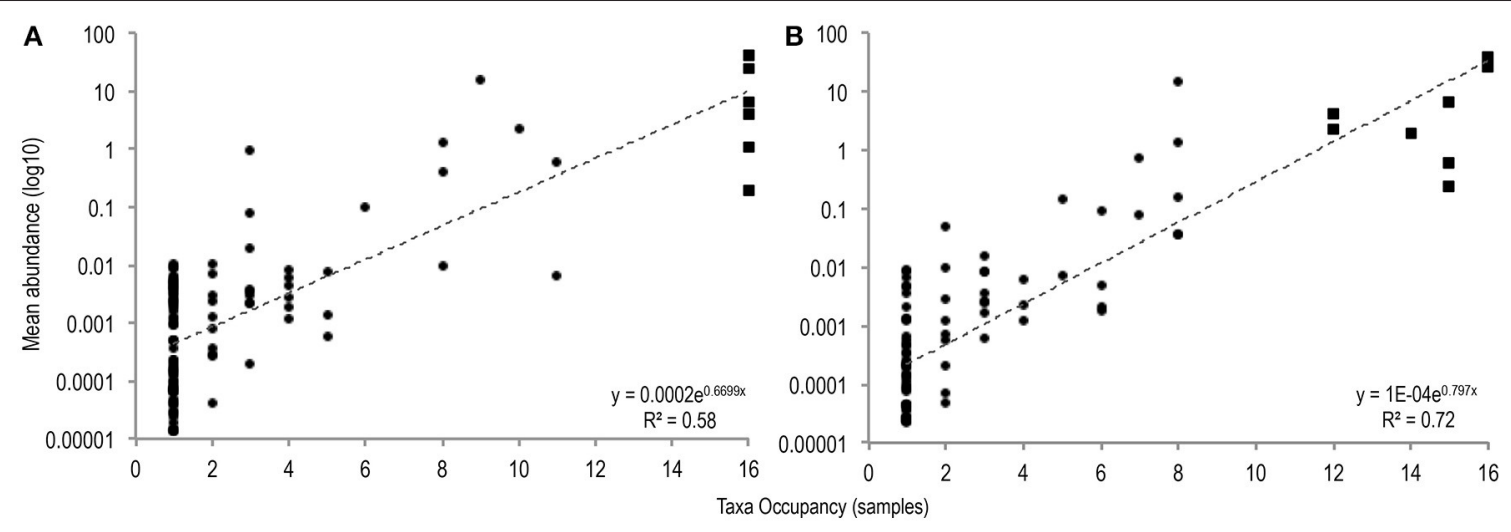

FIGURE 1 | The number of samples in which taxa were observed plotted against mean taxa abundance (log10 scale) in untreated sample (A), and PMA-treated sample (B), conditions [(A: $\left.\left.r^{2}=0.58 ; P=<0.001\right),\left(\mathbf{B}: r^{2}=0.72 ; P=<0.001\right)\right]$. Common taxa $(>75 \%$ sample occupancy) are plotted square, rare samples $(<75 \%$ sample occupancy) are plotted circle.

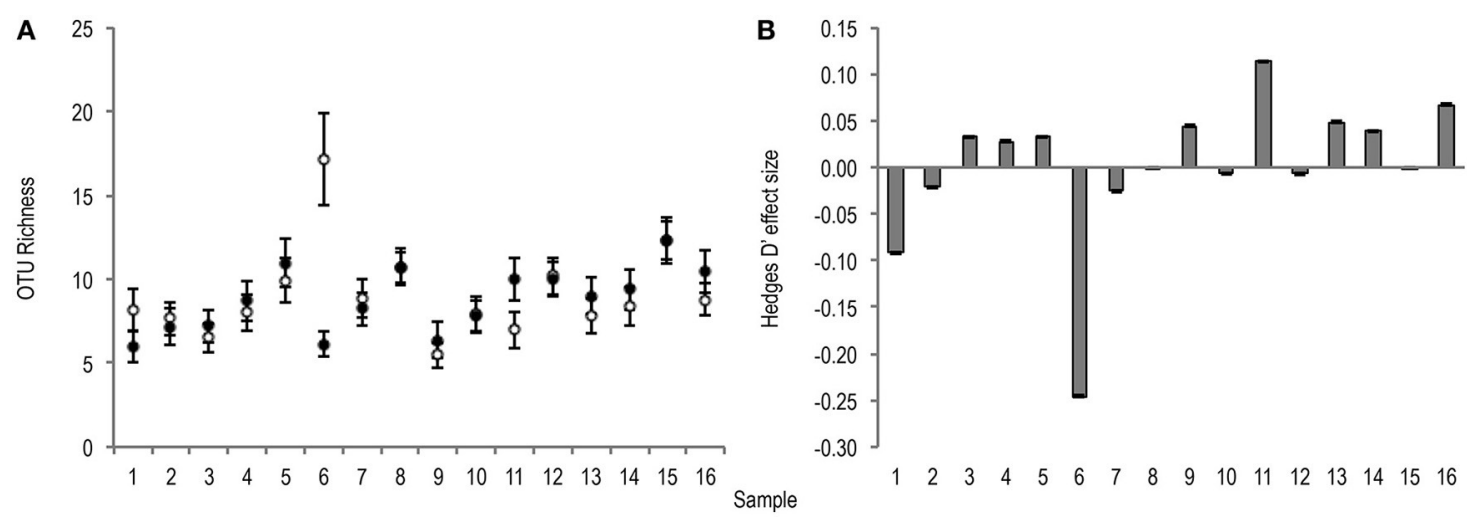

FIGURE 2 | (A) Bootstrapped $(n=1,000) O^{*}$ values for untreated (hollow points), and PMA-treated (solid points) conditions of all samples (1-16). Error bars are included. (B) Hedges' d effect size of PMA-treatment on O* of all samples (1-16). Positive effect was observed in $50 \%$ of samples. Sample 6 was the only observed effect size $>0.2$ (small effect).

TABLE 1 | Mean and standard deviation for Shannon and inverse Simpson diversity indices for PMA-treated and untreated conditions.

\begin{tabular}{|c|c|c|c|c|}
\hline & \multicolumn{4}{|c|}{ Diversity index } \\
\hline & \multicolumn{2}{|c|}{ Shannon (H') } & \multicolumn{2}{|c|}{ Inverse Simpson (1/D) } \\
\hline & Mean & $S D$ & Mean & $S D$ \\
\hline PMA-treated & 1.13 & 0.32 & 2.64 & 0.95 \\
\hline Non-PMA-treated & 1.30 & 0.16 & 2.99 & 0.50 \\
\hline$P$-value & \multicolumn{2}{|c|}{$<0.05$} & \multicolumn{2}{|c|}{$>0.05$} \\
\hline
\end{tabular}

overall effect sizes on both measures of diversity were observed following PMA-treatment ( $\mathrm{m}: \mathrm{H}^{\prime}=-0.95 ; 1 / \mathrm{D}=-1.23$ ).

\section{Effect of PMA-Treatment on Individual Bacterial Taxa Abundance}

To investigate the effect of PMA-treatment on observable abundance values of individual taxa relative sequence abundance was calculated. Initial analysis of the PMA-treatment effect on individual taxa abundance within the bacterial communities of stools was performed by SIMPER (Table 2). SIMPER provides an insight in to the variance, expressed as a percentage, between abundance of taxa from the untreated group and the PMAtreated group.

Table 2 illustrates which taxa contributed greatest to dissimilarity of common and rare community structures between untreated and PMA-treated conditions.

Greater average dissimilarity is observed in rare (83.21\%), than common (42.87), taxa. The taxon labelled Escherichia_shigella by the SILVA database (Quast et al., 2013) appears to contribute to the average dissimilarity between non-PMA and PMA treated conditions most (63.45\%), in spite of a mean abundance difference of only $1.3 \%$. This contrasts with other taxa such as Bifidobacterium and Enterococcus, for which lower average dissimilarities of 15.20 and 12.25 are observed, however much greater mean abundance differences of 14.6 and 14.8 are found, respectively. This incongruence could be explained by variation in abundance of individual taxa between samples within the same condition. The SD of Escherichia 


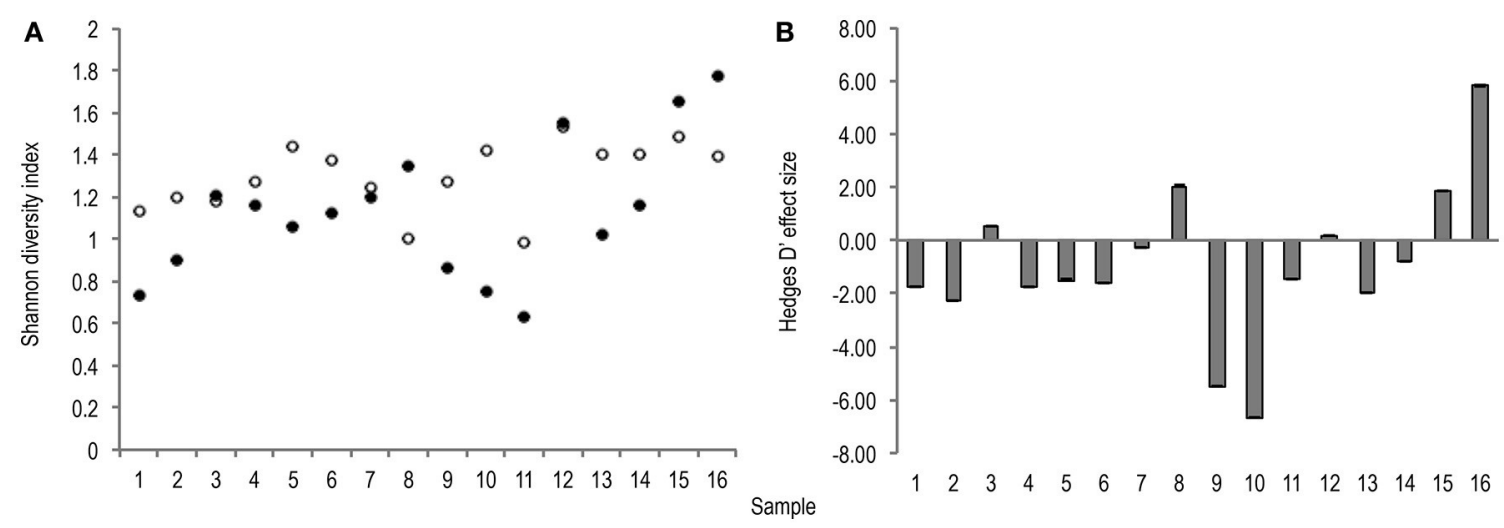

FIGURE 3 | (A) Bootstrapped $(n=1,000)$ Shannon diversity index values for untreated (hollow points), and PMA-treated (solid points), conditions for all samples (1-16). Standard error bars are included. (B) Hedges' d effect size of PMA-treatment on Shannon diversity index values of all samples (1-16). Seventy-five percentage of samples exhibit significant ( $>0.8)$ effect size, $75 \%$ of which are negative.

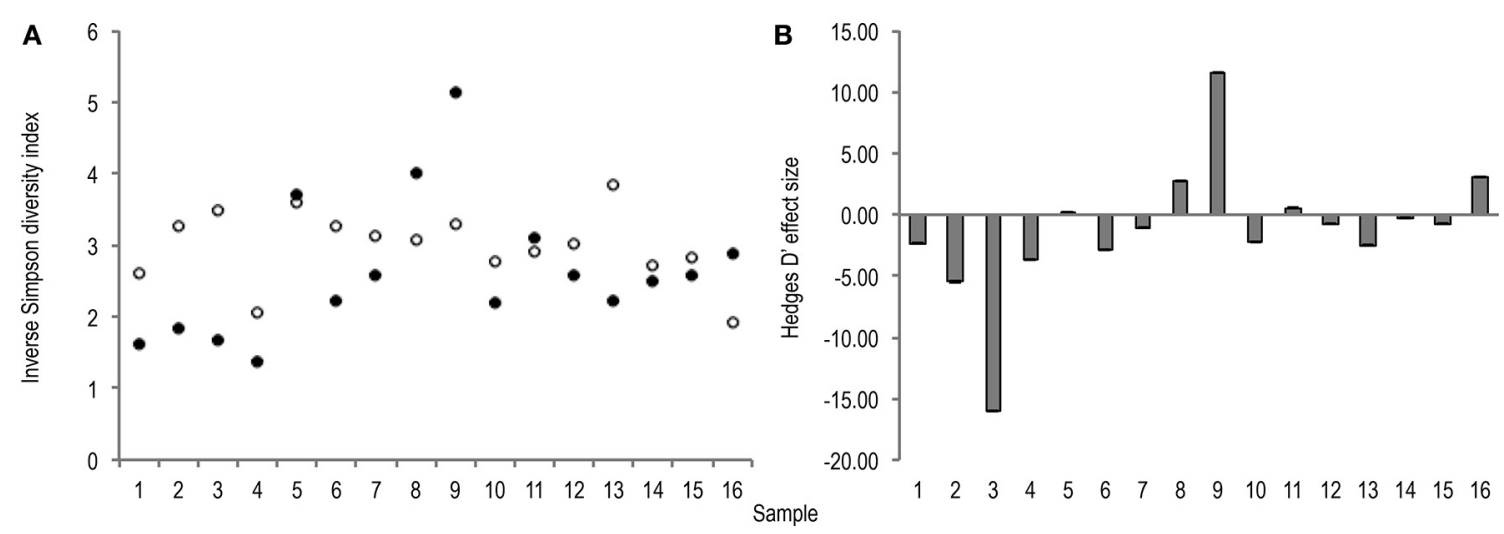

FIGURE 4 | (A) Bootstrapped ( $n=1,000$ ), inverse Simpson diversity index values for untreated (hollow points), and PMA-treated (solid points), conditions for all samples (1-16). Standard error bars are included but error is too insignificant to be visible. (B) Hedges' d effect size of PMA-treatment on inverse Simpson diversity index values of all samples (1-16). 68.8\% samples exhibit significant (>0.8) effect size. $72.3 \%$ of which are negative rare (B) taxa.

abundance is much greater (untreated: $m=16.2, S D=18.2$; PMA-treated: $m=14.9, S D=24.3)$, than that of Bifidobacterium (untreated: $m=41.1, S D=15.2$; PMA-treated: $m=26.5, S D$ $=19.5$ ), or Enterococcus (untreated: $m=25.0, S D=10.2$; PMA-treated: $m=39.8, S D=14.0)$.

To normalise for this variance Wilcoxon rank sum tests were performed to assess the similarities of mean abundance for each taxon in both conditions as identified by SIMPER analysis. The same means were used to calculate a fold change in taxa abundance between the two conditions and both parameters were plotted on a volcano plot (Figure 5).

The majority of taxa showed substantial fold changes in abundance following PMA-treatment; however only 6 of these fold changes pass the significance threshold $(P<0.05)$ : Bifidobacterium; Enterobacteriaceae; Enterococcus; Clostridium; Actinomyces; and Peptoniphilus sp.

In untreated conditions Bifidobacterium and Enterobacteriaceae sp. abundances are significantly greater while Enterococcus, Clostridium, Peptoniphilus, and Actinomyces sp. abundances are significantly lower. This suggests that the presence of non-viable DNA originating from highly abundant species such as Bifidobacteria and Enterobacteriaceae could potentially mask that of less abundant species such as Enterococcus, Clostridium, Peptoniphilus, and Actinomyces sp.

As volcano plots (Figure 5) only represent fold change in abundance for taxa present in both sample conditions, rank abundance plots (Figure 6) were generated to illustrate abundance of taxa identifiable in only one treatment condition.

Fewer taxa were observed PMA-treated than untreated sample conditions. Of 111 total taxa present in only one condition 68 (61.3\%), were present in untreated samples while only 43 (38.7\%), were present in PMA-treated samples, representing a $22.6 \%$ reduction in presence of taxa measurable in only one condition. Levels of 2 rare taxa (Staphylococcus and Phenylobacterium), were observable at levels $>0.09 \%$ sequence abundance (almost 10 fold more than all other taxa observable in only one treatment condition), following PMA-treatment. These taxa were completely masked in the untreated condition. All other 
TABLE 2 | SIMPER analysis of common (A) and rare (B) taxa.

\begin{tabular}{|c|c|c|c|c|c|}
\hline \multicolumn{4}{|c|}{ Average dissimilarity between conditions $=42.87$} & \multirow{2}{*}{$\begin{array}{l}\text { Non-PMA-treated } \\
\text { Mean abund. } 1\end{array}$} & \multirow{2}{*}{$\begin{array}{l}\text { PMA-treated } \\
\text { Mean abund. } 2\end{array}$} \\
\hline Taxon & Av. dissim & Contrib. \% & Cumulative \% & & \\
\hline \multicolumn{6}{|l|}{ (A) } \\
\hline Bifidobacterium & 15.20 & 35.44 & 35.44 & 41.1 & 26.5 \\
\hline Enterococcus & 12.23 & 28.52 & 63.96 & 25.0 & 39.8 \\
\hline Clostridium 1 & 4.60 & 10.74 & 74.70 & 4.0 & 6.6 \\
\hline Enterobacteriaceae; unclassified & 4.03 & 9.41 & 84.11 & 6.6 & 0.6 \\
\hline Anaerococcus* & 3.25 & 7.58 & 91.68 & 2.2 & 4.2 \\
\hline Finegoldia* & 1.75 & 4.08 & 95.76 & 1.3 & 2.3 \\
\hline Clostridium 2 & 1.08 & 2.51 & 98.27 & 0.2 & 1.9 \\
\hline Veillonella & 0.74 & 1.73 & 100.00 & 1.1 & 0.2 \\
\hline \multicolumn{4}{|c|}{ Average dissimilarity between conditions $=\mathbf{8 3 . 2 1}$} & Non-PMA-treated & PMA-treated \\
\hline \multicolumn{6}{|l|}{ (B) } \\
\hline Escherichia_Shigella & 52.81 & 63.46 & 63.46 & 16.2 & 14.9 \\
\hline Peptoniphilus & 11.07 & 13.3 & 76.76 & 0.576 & 1.41 \\
\hline Actinomyces & 6.88 & 8.267 & 85.03 & 0.389 & 0.75 \\
\hline Streptococcus & 4.087 & 4.911 & 89.94 & 0.986 & 0.0157 \\
\hline Staphylococcus & 1.378 & 1.656 & 91.59 & 0 & 0.15 \\
\hline Phenylobacterium & 1.333 & 1.602 & 93.2 & 0 & 0.0939 \\
\hline
\end{tabular}

Cut-off value set at taxa contributing $<1 \%$ to dissimilarity between treatment conditions.

*Denotes taxa only attributed common status in PMA-treated condition.

Cut-off set at taxa contributing $<1 \%$ to dissimilarity between treatment conditions.

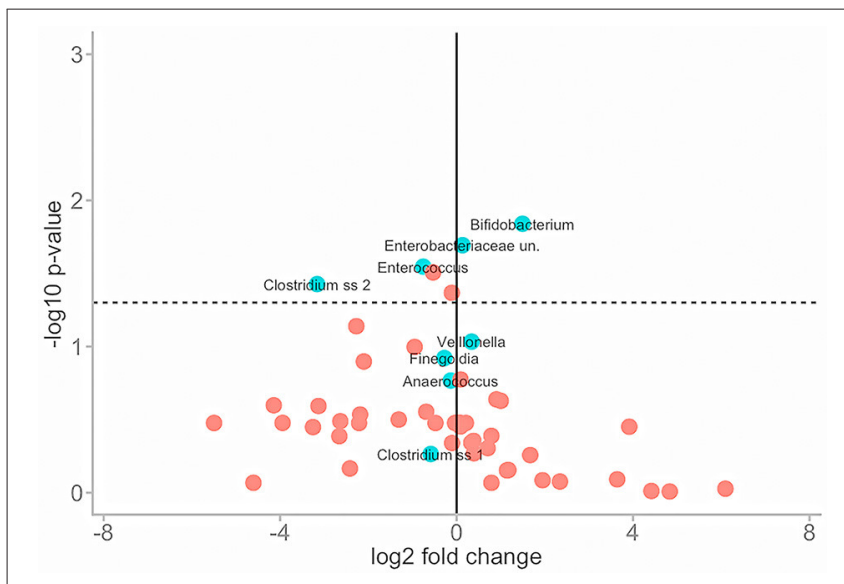

FIGURE 5 | Illustrates the effect of PMA-treatment on individual taxa abundances. Fold change in abundance is plotted $(x)$ against the $P$-value of that same fold change $(y)$ for each taxon. The solid vertical line represents no fold-change. Travelling in either direction from this line the fold change increases. The dotted horizontal line represents significance cut-off (.05). Core taxa are blue and labelled. Rare taxa are red.

taxa abundances were reduced to $<0.009 \%$ following PMAtreatment. A significant difference between mean abundances of rare taxa presence between untreated $(m=0.0052)$, and PMAtreated $(m=0.0012)$, sample conditions $(P=<0.001)$, was observed. This suggests that performing gut microbiota analysis of frozen stool by paired end targeted 16S rRNA gene sequencing without PMA-treatment could fail to identify presence of rare community members due to significant background sequence noise origniating from non-living taxa. This could explain the significant overall reduction in mean bacterial diversity following PMA-treatment observed in meta-analysis (Figures 3, 4). Principle coordinate analysis of sample communities was also performed based on Bray-Curtis dissimilarity of taxa abundances (Figure S2).

To confirm the differences observed were due to genuine viable differences in the sample microbiota rather than bacterial cell death during freezing $\left(-80^{\circ} \mathrm{C}\right)$ one further stool sample was split $(n=10)$. The microbiota of frozen and non-frozen samples in untreated and PMA-treated conditions were compared. Overall $4.04 \times 10^{6}$ reads were recorded from $10 \times 2$ samples $\left(m=2.02 \times 10^{5}\right)$. Sample storage had an insignificant effect on observed Bray-Curtis community similarity between treatment conditions (Table 3), and no separation by PMA-treatment within storage groups was observed (Figure S3). This data further supports Shaw et al. (2016), findings suggesting freeze storage of severely preterm stool samples does not significantly impact the gut microbiota observed with or without PMA-treatment.

\section{DISCUSSION}

The gut microbiota of significantly preterm infants held within the neonatal intensive care unit has been previously identified to be extremely changeable (Koenig et al., 2011; Bergstrom et al., 2014). Microbial communities colonising this biotope are challenged by frequent antibiotic intervention (Craft et al., 2000), administration of probiotics (AlFaleh and Anabrees, 2014), 


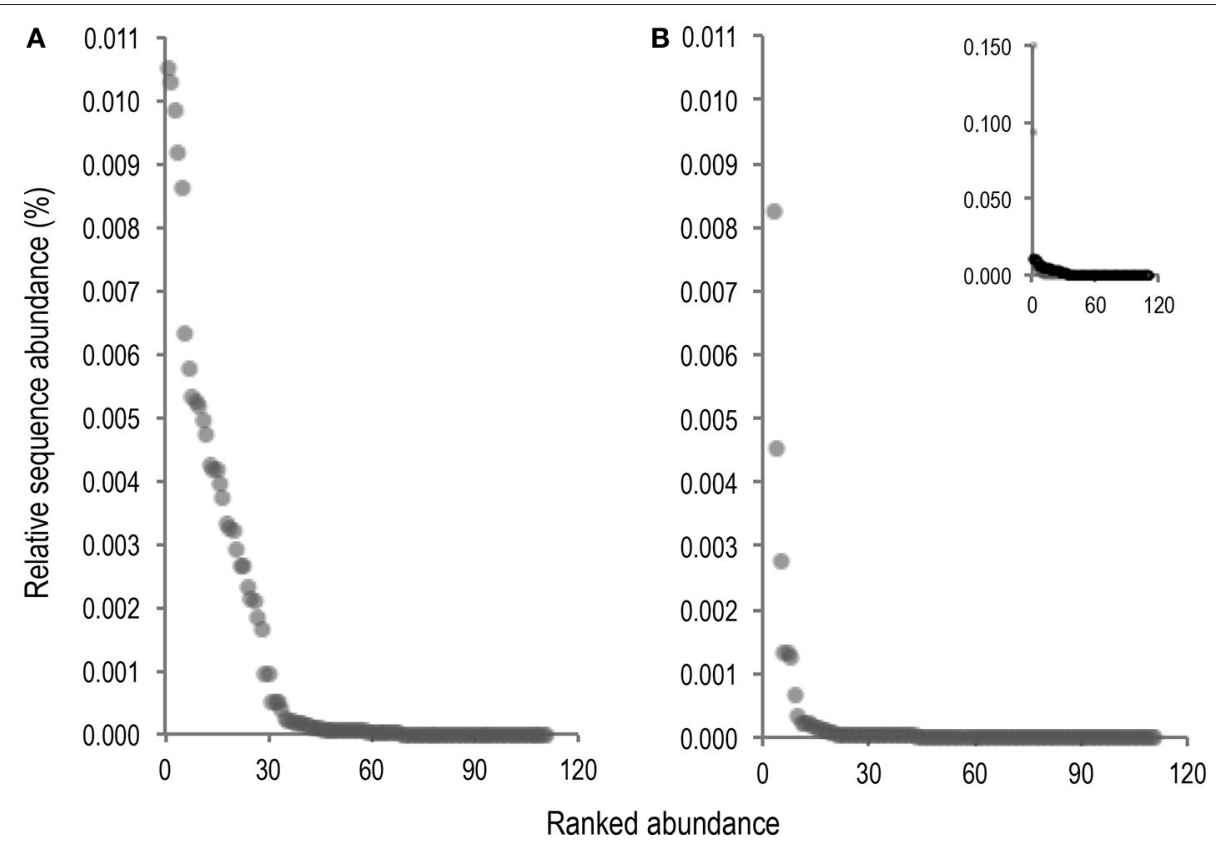

FIGURE 6 | Rank abundance plots of taxa for which abundance was only measurable in either the untreated (A), or PMA-treated (B), sample condition. Ranked abundance of the untreated condition including Staphylococcus and Phenylobacterium is inset at top right corner of PMA-treated condition plot (B).

TABLE 3 | $P$-values associated with ANOSIM analysis comparing Bray Curtis dissimilarity between frozen and non-frozen samples in control and PMA-treated sample conditions.

\begin{tabular}{lcccc}
\hline & \multicolumn{2}{c}{ ANOSIM x groups (Bray-Curtis) } & \\
\cline { 2 - 3 } & $\begin{array}{l}\text { Frozen } \\
\text { CTRL }\end{array}$ & Frozen PMA & $\begin{array}{c}\text { Non-frozen } \\
\text { CTRL }\end{array}$ & $\begin{array}{c}\text { Non-frozen } \\
\text { PMA }\end{array}$ \\
\hline Frozen CTRL & & $>0.1$ & $<0.01$ & $<0.01$ \\
Frozen PMA & $>0.1$ & & $<0.01$ & $<0.01$ \\
Non-frozen CTRL & $<0.01$ & $<0.01$ & & $>0.5$ \\
Non-frozen PMA & $<0.01$ & $<0.01$ & $>0.5$ & \\
\hline
\end{tabular}

fluctuating $\mathrm{pH}$ due to the use of proton pump inhibitors (Omari et al., 2007, 2009), and gut lumen and immune system maturation (Israel, 1994; Levy, 2007; Strunk et al., 2011). All of these factors complex the first months of a newborn infant's life, thereby it is considered the most unstable with respect to microbiota composition. In order for clinicians to accurately assess the requirement for, and effect of, intervention strategies on infant microbial populations the analysis techniques used must be able to reliably quantify unbiased and viable microbiotas.

Currently techniques either cannot provide results within a short turnaround time at a sufficient phylogenetic resolution to assess the diversity in the gut (bacterial culture), or fail to differentiate viable from non-viable community members (QPCR). While RNA sequencing enables exclusive identification of genes actively transcribed by viable cells there are downstream issues regarding storage and contaminating RNAses (Zheng et al., 1996) RNA samples require collection in an RNA preservative (Mutter et al., 2004) which is not always possible in the clinic.
Furthermore, use of DNA in combination with PMA eliminates the need for reverse transcription of sequences prior to analysis.

Nocker and Camper (2009), have previously shown PMAtreatment excludes DNA from non-viable cells. This study builds on those results by illustrating PMA-treatment of frozen preterm infant stool alters observable microbiota structure and diversity following paired end targeted $16 \mathrm{~S}$ rRNA gene sequencing. This would suggest inclusion of non-viable community members during preterm infant stool microbiota analysis introduces a bias. Additionally, DNA from non-viable cells can have significant impact on individual taxa quantification. We propose it may be necessary to employ the use of PMA as a tool for NVCE in 16S rRNA gene sequencing based microbiota analysis. Effects of PMA NVCE should not be attributed to cell death during storage as no difference in PMA effect was observed between frozen and fresh stool samples. It is probable that the changes in abundance illustrated by PMA NVCE are caused by antibiotic, probiotic, or other clinical interventions however further study is required to confirm this.

Importantly, this study illustrates that the presence of particular, clinically relevant taxa may be either over-represented (Bifidobacterium), or under-represented (Clostridium, Staphylococcus), in the absence of PMA-treatment. This is most probably due to the suppression of DNA sequence reads from rare taxa by dominant taxa as illustrated by the reduced bacterial diversity and presence of rare taxa observed in PMA-treated samples.

These findings are of particular relevance in the gut microbiota of the preterm infants analysed in this study due to administration of probiotic supplements. While Bifidobacterium remained ubiquitous and abundant across samples in both 
treatment conditions it has been shown in several studies (Alander et al., 2001; Charbonneau et al., 2013; Rattanaprasert et al., 2014) that administered probiotic strains often fail to engraft long-term. This would make PMA treatment extremely important for analysis of future intervention trials of this manner. Maldonado-Gómez María et al. (2016), showed that presence of phylogenetically or functionally similar keystone species can prevent engraftment of probiotic strains. The results of this study suggest Bifidobacteria within the probiotics may not maintain viability throughout the entire GI tract; a further possible reason for this failed engraftment. We demonstrate persistent DNA from non-viable Bifidobacteria may conceal the presence of less abundant, transient colonisers with the potential to confound clinical inferences drawn from 16S rRNA gene sequencing data. Further work should compare the functional profiles of the probiotic Bifidobacterium strain in Infloran ${ }^{\circledR}$ and the bacterial metagenomes of patients administered the supplement as well as investigating the community engraftment potential of the specific probiotic strains administered to patients enrolled in this study.

This study has deliberately selected a pair of twins with good longitudinal sampling to evaluate the effect of PMA treatment on observable microbiota members, however the number of actual samples $(n=16)$, is relatively small and recruitment purely convenience based. In lieu of the individual taxa for which significant changes in abundance are observed in this study may not be replicated in repeated studies, dependant on viable and non-viable taxa abundances. Specifically, Bifidobacterium may not necessarily be observed at significantly altered abundances in microbiota of patients not receiving probiotics or in patients with a greater engraftment potential. Given the key role of Bifidobacteria in preterm gut health, this requires further exploration. We stress that use of PMA need not be limited to that of preterm infant stool but could be applied to any unstable environment where clinical microbiota intervention is employed or in which abundance of community members may be regularly changeable. Further studies may wish to explore the use of such techniques in these environments.

Consideration should be granted that the use of a nested PCR technique represents a potential source of amplification bias in populations of low bacterial load (Yu et al., 2015). In contrast, Fan et al. (2009), have demonstrated that use of a 25 cycle nested PCR does not significantly affect observable bacterial communities. Moreover, nested PCR was employed in this study to increase the inhibitory capacity of PMA on chelated DNA, rather than increase identifiable sequences.

One possible reason for the widespread disregard of PMA use for NVCE could be the specification of non-viable cells solely as membrane-compromised cells using this method. Contreras et al. (2011), describe membrane integrity as a "conservative parameter" for viability identification, explaining inability to culture bacteria occurs sooner than membrane denaturation in heat-killed cells. We propose conservative NVCE is more appealing in this clinical context than nihil NVCE, in which nonviable DNA persists and can bias results or exaggerated NVCE, where community members may be excluded from analysis while still viable.

This study represents the first time PMA-treatment has been combined with paired end targeted 16S rRNA gene sequence analysis of a gut microbiota using the methods described by Kozich et al. (2013). Future research should focus on validation of this method of analysis in a larger sample cohort to include greater inter-sample microbiota variation. Analysis of probiotic and commensal bacterial viability throughout the preterm infant GI tract would be another logical progression from this work.

\section{ETHICS STATEMENT}

This study was carried out in accordance with the recommendations of "NRES Committee North East - Newcastle \& North Tyneside 2" with written informed consent from all subjects. All subjects gave written informed consent in accordance with the Declaration of Helsinki. The protocol was approved by the "NRES Committee North East - Newcastle \& North Tyneside 2."

\section{AUTHOR CONTRIBUTIONS}

GY, Cv, and CL conceived the study. NE and JB collected samples and clinical data. GY designed the study and performed the experiments. DS ran the sequencing. GY, Cv, ES, and SC analysed the data. GY, DS, and Cv wrote the paper. All authors proof read and approved the paper prior to submission.

\section{FUNDING}

This work was supported by Northumbria University [grant number 10031605/2 awarded to GY].

\section{ACKNOWLEDGMENTS}

The authors would like to acknowledge the work of the clinical staff of the neonatal intensive care unit at the Royal Victoria Infirmary, Newcastle for collection and storage of samples. Particular thanks go to Julie Groombridge, the research nurse at the RVI NICU for organisation and sample labelling. Further personal acknowledgement must be given to Dr. Andrew Nelson, Northumbria University, for being a great antagonist: challenging and questioning the findings; to help improve the validity of the research. Sequencing of the $16 \mathrm{~S}$ rRNA gene was performed by the NUOMICS sequencing service.

\section{SUPPLEMENTARY MATERIAL}

The Supplementary Material for this article can be found online at: http://journal.frontiersin.org/article/10.3389/fcimb. 2017.00237/full\#supplementary-material 


\section{REFERENCES}

Alander, M., Matto, J., Kneifel, W., Johansson, M., Kogler, B., Crittenden, R., et al. (2001). Effect of galacto-oligosaccharide supplementation on human faecal microflora and on survival and persistence of Bifidobacterium lactis $\mathrm{Bb}-12$ in the gastrointestinal tract. Int. Dairy J. 11, 817-825. doi: 10.1016/S0958-6946(01)00100-5

AlFaleh, K., and Anabrees, J. (2014). Probiotics for prevention of necrotizing enterocolitis in preterm infants. Cochrane Database Syst. Rev. 4, 16:CD005496. doi: 10.1002/14651858.CD005496

Bae, S. W., and Wuertz, S. (2009). Discrimination of viable and dead fecal bacteroidales bacteria by quantitative PCR with propidium monoazide. Appl. Environ. Microbiol. 75, 2940-2944. doi: 10.1128/AEM.013 $33-08$

Banihashemi, A., Van Dyke, M. I., and Huck, P. M. (2012). Longamplicon propidium monoazide-PCR enumeration assay to detect viable Campylobacter and Salmonella. J. Appl. Microbiol. 113, 863-873. doi: 10.1111/j.1365-2672.2012.05382.x

Bergstrom, A., Skov, T. H., Bahl, M. I., Roager, H. M., Christensen, L. B., Ejlerskov, K. T., et al. (2014). Establishment of intestinal microbiota during early life: a longitudinal, explorative study of a large cohort of danish infants. Appl. Environ. Microbiol. 80, 2889-2900. doi: 10.1128/AEM.00342-14

Boccia, D., Stolfi, I., Lana, S., and Moro, M. L. (2001). Nosocomial necrotising enterocolitis outbreaks: epidemiology and control measures. Eur. J. Pediatr. 160, 385-391. doi: 10.1007/s004310100749

Borenstein, M., Hedges, L. V., Higgins, J. P. T., and Rothstein, H. R. (2009). How a Meta-Analysis Works. Chichester: John Wiley \& Sons, Ltd.

Caporaso, J. G., Lauber, C. L., Walters, W. A., Berg-Lyons, D., Huntley, J., Fierer, N., et al. (2012). Ultra-high-throughput microbial community analysis on the Illumina HiSeq and MiSeq platforms. ISME J. 6, 1621-1624. doi: 10.1038 /ismej.2012.8

Caporaso, J. G., Lauber, C. L., Walters, W. A., Berg-Lyons, D., Lozupone, C. A., Turnbaugh, P. J., et al. (2011). Global patterns of $16 \mathrm{~S}$ rRNA diversity at a depth of millions of sequences per sample. Proc. Natl. Acad. Sci. U.S.A. 108, 4516-4522. doi: 10.1073/pnas.1000080107

Charbonneau, D., Gibb, R. D., and Quigley, E. M. (2013). Fecal excretion of Bifidobacterium infantis 35624 and changes in fecal microbiota after eight weeks of oral supplementation with encapsulated probiotic. Gut. Microbes 4, 201-211. doi: 10.4161/gmic.24196

Contreras, P. J., Urrutia, H., Sossa, K., and Nocker, A. (2011). Effect of PCR amplicon length on suppressing signals from membrane-compromised cells by propidium monoazide treatment. J. Microbiol. Methods 87, 89-95. doi: 10.1016/j.mimet.2011.07.016

Craft, A. P., Finer, N. N., and Barrington, K. J. (2000). Vancomycin for prophylaxis against sepsis in preterm neonates. Cochrane Database Syst. Rev. 2:CD001971. doi: 10.1002/14651858.CD001971

Fan, Z.-Y., Li, X.-R., Mao, D.-P., Zhu, G.-F., Wang, S.-Y., and Quan, Z.-X. (2009). Could nested PCR be applicable for the study of microbial diversity? World J. Microbiol. Biotechnol. 25, 1447-1452. doi: 10.1007/s11274-0090033-3

Fanaro, S. (2013). Feeding intolerance in the preterm infant. Early Hum. Dev. 89(Suppl. 2), S13-S20. doi: 10.1016/j.earlhumdev.2013.07.013

Fujimoto, J., and Watanabe, K. (2013). Quantitative detection of viable Bifidobacterium bifidum BF-1 Cells in human feces by using propidium monoazide and strain-specific primers. Appl. Environ. Microbiol. 79, 2182-2188. doi: 10.1128/AEM.03294-12

Gregory, K. E., Deforge, C. E., Natale, K. M., Phillips, M., and Van Marter, L. J. (2011). Necrotizing enterocolitis in the premature infant: neonatal nursing assessment, disease pathogenesis, and clinical presentation. Adv. Neonatal Care 11, 155-164. doi: 10.1097/ANC.0b013e31821baaf4

Halpern, M. D., and Denning, P. W. (2015). The role of intestinal epithelial barrier function in the development of NEC. Tissue Barriers 3:e1000707. doi: 10.1080/21688370.2014.1000707

Hammer, Ø., Harper, D. A. T., and Ryan, P. D. (2001). "Past: Paleontological Statistics Software Package for education and data analysis," in Palaeontologia Electronica, Vol. 4, 9. Available online at: http://palaeo-electronica.org/2001_1/ past/issue1_01.htm
Hoy, C. M., Wood, C. M., Hawkey, P. M., and Puntis, J. W. L. (2000). Duodenal microflora in very-low-birth weight neonates and relation to necrotizing enterocolitis. J. Clin. Microbiol. 38, 4539-4547. Available online at: https://www.scopus.com/record/display.uri?eid=2-s2.0-0034460430\&origin= inward\&txGid=A4A23F490D36796B4CB5E0ED1D87F2CE.wsnAw8kcdt7IPY LO0V48gA\%3a1

Israel, E. J. (1994). Neonatal necrotizing enterocolitis, a disease of the immature intestinal Mucosal Barrier. Acta Paediatr. 83, 27-32. doi: 10.1111/j.1651-2227.1994.tb13238.x

Koenig, J. E., Spor, A., Scalfone, N., Fricker, A. D., Stombaugh, J., Knight, R., et al. (2011). Succession of microbial consortia in the developing infant gut microbiome. Proc. Natl. Acad. Sci. U.S.A. 108, 4578-4585. doi: $10.1073 /$ pnas. 1000081107

Kozich, J. J., Westcott, S. L., Baxter, N. T., Highlander, S. K., and Schloss, P. D. (2013). Development of a dual-index sequencing strategy and curation pipeline for analyzing amplicon sequence data on the MiSeq illumina sequencing platform. Appl. Environ. Microbiol. 79, 5112-5120. doi: 10.1128/AEM.01043-13

Lane, D. J. (1991). "16S/23S rRNA sequencing," in Nucleic Acid Techniques in Bacterial Systematics, eds E. Stackebrandt and M Goodfellow (Chichester, UK: John Wiley \& Sons), 115-175.

Leff, L. G., Dana, J. R., McArthur, J. V., and Shimkets, L. J. (1995). Comparison of methods of DNA extraction from stream sediments. Appl. Environ. Microbiol. 61, 1141-1143.

Levy, O. (2007). Innate immunity of the newborn: basic mechanisms and clinical correlates. Nat. Rev. Immunol. 7, 379-390. doi: 10.1038/nri2075

Mai, V., Young, C. M., Ukhanova, M., Wang, X. Y., Sun, Y. J., Casella, G., et al. (2011). Fecal microbiota in premature infants prior to necrotizing Enterocolitis. PLoS ONE 6:e20647. doi: 10.1371/journal.pone.0020647

Maldonado-Gómez María, X., Martínez, I., Bottacini, F., O’Callaghan, A., Ventura, M., van Sinderen, D., et al. (2016). Stable engraftment of Bifidobacterium longum AH1206 in the human gut depends on individualized features of the resident microbiome. Cell Host Microbe 4, 515-526. doi: 10.1016/j.chom.2016.09.001

McMurty, V. E., Gupta, R. W., Tran, L., Blanchard, E. E., Penn, D., Taylor, C. M., et al. (2015). Bacterial diversity and Clostridia abundance decrease with increasing severity of necrotizing enterocolitis. Microbiome 3, 11. doi: 10.1186/s40168-015-0075-8

Mshvildadze, M., Neu, J., Shuster, J., Theriaque, D., Li, N., and Mai, V. (2010). Intestinal microbial ecology in premature infants assessed with non-culturebased techniques. J. Pediatr. 156, 20-25. doi: 10.1016/j.jpeds.2009.06.063

Mutter, G. L., Zahrieh, D., Liu, C., Neuberg, D., Finkelstein, D., Baker, H. E., et al. (2004). Comparison of frozen and RNALater solid tissue storage methods for use in RNA expression microarrays. BMC Gen. 5:88. doi: 10.1186/1471-2164-5-88

Nguyen, L. D. N., Deschaght, P., Merlin, S., Loywick, A., Audebert, C., Van Daele, S., et al. (2016). Effects of Propidium Monoazide (PMA) treatment on mycobiome and bacteriome analysis of cystic fibrosis airways during exacerbation. PLoS ONE 11:e0168860. doi: 10.1371/journal.pone.0168860

Nocker, A., and Camper, A. K. (2009). Novel approaches toward preferential detection of viable cells using nucleic acid amplification techniques. FEMS Microbiol. Lett. 291, 137-142. doi: 10.1111/j.1574-6968.2008. 01429.x

Nocker, A., Cheung, C. Y., and Camper, A. K. (2006). Comparison of propidium monoazide with ethidium monoazide for differentiation of live vs. dead bacteria by selective removal of DNA from dead cells. J. Microbiol. Methods 67, 310-320. doi: 10.1016/j.mimet.2006.04.015

Nocker, A., Richter-Heitmann, T., Montijn, R., Schuren, F., and Kort, R. (2010). Discrimination between live and dead cells in bacterial communities from environmental water samples analyzed by 454 pyrosequencing. Int. Microbiol. 13, 59-65. doi: 10.2436/20.1501.01.111

Nocker, A., Sossa-Fernandez, P., Burr, M. D., and Camper, A. K. (2007). Use of propidium monoazide for live/dead distinction in microbial ecology. Appl. Environ. Microbiol. 73, 5111-5117. doi: 10.1128/AEM.02987-06

Oksanen, J., Guillaume Blanchet, F., Kindt, R., Legendre, P., Minchin, P. R., O'Hara, R. B., et al. (2015). vegan: Community Ecology Package.

Omari, T. I., Haslam, R. R., Lundborg, P., and Davidson, G. P. (2007). Effect of omeprazole on acid gastroesophageal reflux and gastric acidity in preterm 
infants with pathological acid reflux. J. Pediatr. Gastroenterol. Nutr. 44, 41-44. doi: 10.1097/01.mpg.0000252190.97545.07

Omari, T., Lundborg, P., Sandstrom, M., Bondarov, P., Fjellman, M., Haslam, R., et al. (2009). Pharmacodynamics and systemic exposure of esomeprazole in preterm infants and term neonates with gastroesophageal reflux disease. $J$. Pediatr. 155, 222-228. doi: 10.1016/j.jpeds.2009.02.025

Quast, C., Pruesse, E., Yilmaz, P., Gerken, J., Schweer, T., Yarza, P., et al. (2013). The SILVA ribosomal RNA gene database project: improved data processing and web-based tools. Nucl. Acids Res. 41, D590-D596. doi: 10.1093/nar/gks1219

R_Core_Team. (2014). R: A Language and Environment for Statistical Computing. R Foundation for Statistical Computing. Available online at: http://www.R-project.org/

Rattanaprasert, M., Roos, S., Hutkins, R. W., and Walter, J. (2014). Quantitative evaluation of synbiotic strategies to improve persistence and metabolic activity of Lactobacillus reuteri DSM 17938 in the human gastrointestinal tract. J. Funct. Foods 10, 85-94. doi: 10.1016/j.jff.2014.05.017

Rochelle, P. A., Cragg, B. A., Fry, J. C., Parkes, R. J., and Weightman, A. J. (1994). Effect of sample handling on estimation of bacterial diversity in marine sediments by $16 \mathrm{~S}$ rRNA gene sequence analysis. FEMS Microbiol. Ecol. 15, 215-225. doi: 10.1111/j.1574-6941.1994.tb00245.x

Rogers, G. B., Cuthbertson, L., Hoffman, L. R., Wing, P. A. C., Pope, C., Hooftman, D. A. P., et al. (2013). Reducing bias in bacterial community analysis of lower respiratory infections. ISME J. 7, 697-706. doi: 10.1038/ismej.2012.145

Rogers, G. B., Marsh, P., Stressmann, A. F., Allen, C. E., Daniels, T. V. W., Carroll, M. P., et al. (2010). The exclusion of dead bacterial cells is essential for accurate molecular analysis of clinical samples. Clin. Microbiol. Infect. 16, 1656-1658. doi: 10.1111/j.1469-0691.2010.03189.x

Sanchez, M. C., Marin, M. J., Figuero, E., Llama-Palacios, A., Herrera, D., and Sanz, M. (2013). Analysis of viable vs. dead Aggregatibacter actinomycetemcomitans and Porphyromonas gingivalis using selective quantitative real-time PCR with propidium monoazide. J. Periodontal Res. 48, 213-220. doi: 10.1111/j.1600-0765.2012.01522.x

Schloss, P. D., and Westcott, S. L. (2011). Assessing and Improving methods used in operational taxonomic unit-based approaches for 16S rRNA gene sequence analysis. Appl. Environ. Microbiol. 77, 3219-3226. doi: 10.1128/AEM.02810-10

Schloss, P. D., Westcott, S. L., Ryabin, T., Hall, J. R., Hartmann, M., Hollister, E. B., et al. (2009). Introducing mothur: open-source, platformindependent, community-supported software for describing and comparing microbial communities. Appl. Environ. Microbiol. 75, 7537-7541. doi: 10.1128/AEM.01541-09

Schneegurt, M. A., Dore, S. Y., and Kulpa, C. F. Jr. (2003). Direct extraction of DNA from soils for studies in microbial ecology. Curr. Issues Mol. Biol. 5, 1-8.

Shaw, A. G., Sim, K., Powell, E., Cornwell, E., Cramer, T., McClure, Z. E., et al. (2016). Latitude in sample handling and storage for infant faecal microbiota studies: the elephant in the room? Microbiome 4, 1-14. doi: 10.1186/s40168-016-0186-x
Stoll, B. J., Gordon, T., Korones, S. B., Shankaran, S., Tyson, J. E., Bauer, C. R., et al. (1996). Early-onset sepsis in very low birth weight neonates. A report from the National Institute of Child Health and Human Development Neonatal Research Network. J. Pediatr. 129, 72-80. doi: 10.1016/S0022-3476(96)70192-0

Strunk, T., Currie, A., Richmond, P., Simmer, K., and Burgner, D. (2011). Innate immunity in human newborn infants: prematurity means more than immaturity. J. Matern. Fetal Neonatal Med. 24, 25-31. doi: 10.3109/14767058.2010.482605

Torrazza, R. M., Ukhanova, M., Wang, X. Y., Sharma, R., Hudak, M. L., Neu, J., et al. (2013). Intestinal Microbial ecology and environmental factors affecting necrotizing Enterocolitis. PLoS ONE 8:e83304. doi: 10.1371/journal.pone.0083304

Tuddenham, S., and Sears, C. L. (2015). The intestinal microbiome and health. Curr. Opin. Infect. Dis. 28, 464-470. doi: 10.1097/QCO.0000000000000196

Turner, S., Pryer, K. M., Miao, V. P. W., and Palmer, J. D. (1999). Investigating deep phylogenetic relationships among cyanobacteria and plastids by small subunit rRNA sequence analysis1. J. Eukaryot. Microbiol. 46, 327-338. doi: 10.1111/j.1550-7408.1999.tb04612.x

von Wintzingerode, F., Gobel, U. B., and Stackebrandt, E. (1997). Determination of microbial diversity in environmental samples: pitfalls of PCR-based rRNA analysis. FEMS Microbiol. Rev. 21, 213-229. doi: 10.1111/j.1574-6976.1997.tb00351.x

Wang, G. C. Y., and Wang, Y. (1996). The frequency of chimeric molecules as a consequence of PCR co amplification of $16 \mathrm{~S}$ rRNA genes from different bacterial species. Microbiology 142, 1107-1114. doi: 10.1099/13500872-142-5-1107

Weinstock, G. M. (2012). Genomic approaches to studying the human microbiota. Nature 489, 250-256. doi: 10.1038/nature11553

Yu, G., Fadrosh, D., Goedert, J. J., Ravel, J., and Goldstein, A. M. (2015). Nested PCR Biases in interpreting microbial community structure in 16S rRNA gene sequence datasets. PLoS ONE 10:e0132253. doi: 10.1371/journal.pone.0132253

Zheng, D., Alm, E. W., Stahl, D. A., and Raskin, L. (1996). Characterization of universal small-subunit rRNA hybridization probes for quantitative molecular microbial ecology studies. Appl. Environ. Microbiol. 62, 4504-4513.

Conflict of Interest Statement: The authors declare that the research was conducted in the absence of any commercial or financial relationships that could be construed as a potential conflict of interest.

Copyright (c) 2017 Young, Smith, Embleton, Berrington, Schwalbe, Cummings, van der Gast and Lanyon. This is an open-access article distributed under the terms of the Creative Commons Attribution License (CC BY). The use, distribution or reproduction in other forums is permitted, provided the original author(s) or licensor are credited and that the original publication in this journal is cited, in accordance with accepted academic practice. No use, distribution or reproduction is permitted which does not comply with these terms. 